\title{
El efecto del ciclo económico en la estructura de las provisiones bancarias para créditos incobrables: Caso Ecuador 2004-2016
}

\author{
The effect of the economic cycle on the structure of bank provisions for bad loans: Case \\ of Ecuador 2004-2016
}

\author{
Patricio Mendieta Andrade \\ Universidad Católica de Cuenca \\ pmendietaa@ucacue.edu.ec
}

\section{Resumen}

El presente artículo analiza la relación existente entre la evolución de las provisiones para cuentas incobrables realizadas por los bancos conformantes del sistema financiero ecuatoriano y el ciclo económico, expresado como los cambios del Producto Interno Bruto (PIB). En el análisis correlacional de un conjunto de datos que comprende el periodo 2004-2016, se identifica el efecto de la inclusión, vía normativa, de la provisión anticíclica en la estructura de ésta cuenta, desatacándose dos escenarios diferentes; uno previo a la norma, en el cual se verifica la relación contracíclica entre la variación del PIB y las provisiones y un segundo escenario, posterior a la vigencia de la norma, donde es posible apreciar un cambio en la relación entre las variables estudiadas, y comprobándose la significancia que adquiere el crecimiento de la cartera en la explicación de la variación de las provisiones.

Palabras claves: Provisiones bancarias, ciclo económico, correlación

Código JEL: E32, E51, G21, G28

\begin{abstract}
The present article analyzes the relationship between the evolution of the provisions for bad accounts made by the banks conforming to the Ecuadorian financial system and the change in the economic cycle, expressed as changes in the Gross Domestic Product (GDP). In the correlation analysis of a data set that covers the period 2004-2016, the effect of the normative inclusion of the countercyclical provision in the structure of this account is identified, uncovering two different scenarios; One prior to the norm, in which the countercyclical relationship between the variation of the GDP and the provisions is verified, and a second scenario, after the validity of the norm, where it is possible to appreciate a change in the relationship between the variables studied, and Being verified the significance that the growth of the portfolio acquires in the explanation of the variation of the provisions.
\end{abstract}

Keywords: Banking provisions, business cycle, correlation

JEL code: E32, E51, G21, G28

\section{Introducción}

A lo largo de la historia moderna, los economistas han realizado esfuerzos para identificar la incidencia que tienen las fluctuaciones macroeconómicas sobre el comportamiento de los diferentes segmentos de la sociedad. En este sentido, ha sido importante el estudio de la 
interacción entre los bancos y la marcha económica de las naciones; siendo importante analizar las variaciones en el crecimiento de la economía y sus efectos en el sector bancario; identificando la incidencia de los factores macroeconómicos en el rol que han desempeñado los bancos captando el ahorro público y orientándolo a los destinos correspondientes, de conformidad con los incentivos y tendencias del mercado (Seffino, m \& Hoyos,D, 2016).

En el contexto anterior, las instituciones bancarias asumen riesgos en sus operaciones crediticias; las cuales, llevan implícito cierto nivel de incertidumbre, el mismo que debe ser cuantificado, a fin de tomar las decisiones orientadas a optimizar el uso y minimizar la exposición de los activos a los diferentes riesgos. Esta reducción de la exposición al riesgo se basa en criterios sustentados en las expectativas y el análisis de la información para realizar procesos racionales previos a la toma decisiones (Zepeda, E., Leos, J. A., \& Carvallo, F. 2016).

Para Bushman y Williams (2012), el aprovisionamiento contra pérdidas en préstamos constituye una opción contable directamente influenciada por la volatilidad y aspectos cíclicos de los resultados bancarios; más se puntualiza que la normativa general de provisiones refuerza los efectos procíclicos de la regulación del capital, dado el carácter ex post del reconocimientos de las pérdidas crediticias, por lo cual recomiendan los autores, adoptar una visión hacia el futuro (ex ante) al momento de estructurar las provisiones a fin de amortiguar dicha prociclicidad.

Este trabajo investigativo analizó la relación existente entre la estructura de las provisiones para cuentas incobrables realizadas por los bancos conformantes del sistema financiero ecuatoriano y la evolución del PIB nacional durante el período 2004- 2016. Con este fin se analizó los saldos de las cuentas de provisiones en el período especificado a fín de evaluar el grado de correlación entre la evolución de las mismas, como variable dependiente, y se incluyó como variable explicativa la variación del PIB.

De lo expuesto y adicional a esta sección introductoria, los resultados de este trabajo investigativo se exponen en cuatro secciones: adicionales: en una segunda sección se pone en relieve los aspectos más sobresalientes del marco teórico bajo el cual se analizan los temas aquí planteados, en la sección 3 se explica la metodología utilizada para el análisis propuesto, la sección 4 muestra los resultados obtenidos y el análisis de los mismos; finalmente, se desarrollan las conclusiones de nuestro análisis.

\section{MARCO TEÓRICO}

\section{Las Provisiones bancarias para créditos incobrables y su marco regulatorio}

El Código Orgánico Monetario y Financiero (2014) especifica que las actividades financieras representan el conjunto de operaciones y servicios que se efectúan entre oferentes, demandantes y usuarios, facilitando la circulación de dinero y realizando la intermediación financiera. El citado Código especifica que, dentro de las operaciones pasivas, las entidades financieras podrán otorgar préstamos, estando obligadas a reflejar la verdadera calidad de estos activos; siendo éstas operaciones bancarias monitoreadas por los estados, Zhen-Jia-Liu (2015) destaca el papel de la Federal Deposit Insurance Corporation (FDIC) en el caso de los EEUU, en el monitoreo de la calidad de los activos, haciendo referencia éste control a la evaluación de los deudores para pagar los préstamos. 
En referencia a lo anotado, la Superintendencia de Bancos del Ecuador dentro de su normativa, específicamente en el Libro I: Normas Generales para las Instituciones del Sistema Financiero, en el Título IX: De los Activos y de los Límites de Crédito (Superintendencia de Bancos, 2016) establece la obligatoriedad de los bancos de efectuar un seguimiento permanente a los activos de riesgo, y determinar el saldo mínimo de provisiones para cuentas incobrables. En este sentido, Ruiz (2008), anota que las provisiones de activo, vistas como la depreciación en el valor contable de activos fijos, existencias, cuentas por cobrar e inversiones financieras temporales, se enmarcan al nuevo concepto de pérdidas por deterioro contemplado en las NIIF.

En el plano nacional ecuatoriano, el Plan de Cuentas de la Superintendencia de Bancos, establece que "la cuenta Provisiones para créditos incobrables es una cuenta de valuación del activo" (Superintendencia de Bancos, 20116, p.58). El citado documento especifica que cada institución financiera podrá constituir provisiones con el objetivo de cubrir potenciales pérdidas basándose en su propia experiencia. En el Ecuador, como se verá más adelante, antes del 2012 se distinguían dos tipos de provisiones para créditos incobrables: la primera de tipo específico constituida para cubrir pérdidas por activos de carácter dudosos según sea pertinente en cada caso concreto y la otra de tipo genérico que se constituye, a través de un porcentaje fijo al activo que se podrían decir sanos (Saurina, 2002).

La Superintendencia de Bancos (2016) especifica que aspectos como el tipo de crédito, determinarán el correspondiente riesgo y su calificación; siendo que, en el Libro I: Normas Generales para las Instituciones financieras, Título IX: De la Gestión y Administración de Riesgo define al riesgo de crédito como "la posibilidad de pérdida debido al incumplimiento del prestatario que conlleva el no pago, el pago parcial o la falta de oportunidad en el pago de las obligaciones pactadas" (Superintendencia de Bancos, 2016, p.1). De este modo, el principal riesgo que afecta al sector bancario es el que sus deudores pierdan la capacidad de pago (total o parcial) de las obligaciones comprometidas (Jara, 2005).

En línea con lo anotado, los autores Hilbers, Otker-Robe, \& Pazarbasioglu, (2006) destacan la importancia del análisis del efecto de las fluctuaciones macroeconómicas en el comportamiento del deudor, destacando la existencia de una correlación entre las variables económicas a nivel macro y el cumplimiento de las obligaciones bancarias. En este sentido, Borio citado por Jara (2005) especifica que el riesgo crediticio sufre una acumulación en los períodos de expansión económica, dada la expansión de la cartera de créditos de los bancos y que, en períodos recesivos, se evidencia la materialización del incumplimiento en los pagos de los créditos, lo cual no implica necesariamente un crecimiento del riesgo crediticio.

En la misma línea de análisis, Sánchez (2008) destaca que las carteras de créditos de las entidades financieras reaccionan de diferente manera dependiendo de la fase del ciclo económico en la que se encuentra la economía nacional; así, se observa un alto crecimiento de los préstamos y un bajo nivel de provisiones durante las fase de expansión económica, mientras que en la fase contractiva del ciclo se evidencia un racionamientos de los préstamos y el aumento en el porcentaje de las provisiones, dada el hecho de que en la primera fase los prestatarios aparentan una buena calidad en su pago y por lo tanto los bancos reducen sus provisiones, ocurriendo lo contrario en la fase de contracción. El autor citado concluye que, esta tendencia se justifica por el hecho de que, en épocas de crecimiento económico el optimismo de los bancos sobre las expectativas de sus prestatarios crece, sumado al crecimiento de los ahorros y una posición sólida de los bancos (mayor capital del requerido), dentro de un ambiente competitivo exacerbado, ambiente propicio para políticas de 
evaluación de riesgo más liberales y consecuentemente una reducción de los niveles de provisiones.

\section{Las Provisiones anticíclicas}

Teniendo como antecedente lo expuesto, en el año 2012 con la Resolución No JB2012-2217de la Superintendencia de Bancos (2012) se establece la Provisión Anticíclica, con el objetivo de lograr una mayor cobertura al riesgo crediticio y así fortalecer la solvencia de los bancos ecuatorianos, realizando la siguiente puntualización:

Provisión anticíclica es aquella que permite contrarrestar el excesivo perfil cíclico de la provisión específica y genérica, por medio de la creación de un fondo para insolvencias durante la fase expansiva, en la que aumenta el riesgo latente (pérdida esperada).

El objetivo de una provisión de este tipo es crear un fondo para posibles insolvencias durante la fase de expansión de la economía, en la que aumenta el riesgo latente; más tarde, en el curso del ciclo económico, ya en la fase de recesión suben los índices de morosidad, siendo esta la manifestación ex post del riesgo crediticio, mas su impacto sobre los resultados y la solvencia es menor al haber sido provisionada con antelación mediante este tipo de provisiones anticíclicas (Salas, 2002).

Se podría concluir que con la constitución de la ya señalada provisión anticíclica se conforma un fondo provisional, el cual se acumula en tanto la pérdida latente supere a las provisiones para créditos incobrables, en los períodos de crecimiento de la cartera, el cual debería ser coincidente con el crecimiento de la economía. Por otro lado, en épocas de contracción, donde el saldo de la cuenta provisiones para cuentas incobrables sea superior a la perdida latente, esta diferencia sería cubierta por el citado fondo provisional (Superintendencia de Bancos, 2012).

\section{METODOLOGÍA}

Para la presente investigación se analizaron los datos disponibles en los Boletines Financieros de la Superintendencia de Bancos del Ecuador (2017), en base a los estados financieros mensuales remitidos por las Instituciones bajo su control, en el período enero 2004 hasta diciembre de 2016, siendo analizados los saldos de las cuentas al último día de cada trimestre.

Por otro lado, al realizar el análisis del ciclo económico se utilizó la información correspondiente a las variaciones macroeconómicas contenidas en el Boletín de Cuentas Nacionales Trimestral del Ecuador expuesto en la página web institucional del Banco Central Ecuatoriano (2017). Con este propósito se analizó la variación entre trimestres del PIB a su valor nominal, con el fin de poder contrastarlo con los saldos expresados en los balances de los bancos y encontrar posibles correlaciones.

Cabe anotar, que en este trabajo se valora la asociación entre variables cuantitativas analizando el grado de correlación existente entre éstas, para lo cual se utilizó el coeficiente de correlación de Pearson; el cual, describe la fuerza de la relación entre dos o más conjuntos de variables en una escala de intervalo o razón. Dicho coeficiente oscila entre -1 y +1 ; determinándose una correlación perfecta mientras el coeficiente calculado se acerca a la unidad (Pita, \& Pértega, 1997).

Un aspecto importante que se analizó fue el impacto de la inclusión, mediante norma, de la provisión anticíclica; para lo cual se subdividió el espacio de tiempo de estudio en dos 
sub-períodos, el primero corresponde a los datos precedentes al tercer trimestre del 2012 y el segundo a los trimestres posteriores, con el objetivo de contrastar el análisis correlacional entre las variables estudiadas, y evaluar la existencia de un cambio en el efecto del PIB en las provisiones para créditos incobrables.

\section{RESULTADOS Y DISCUSIÓN}

Como punto de partida de nuestro análisis, en la Tabla 1 se reconoce la relación existente entre la variación de la cartera $\left(\mathrm{VAR}_{\mathrm{CAR}}\right)$ y la variación del PIB (VAR $\mathrm{PIB}_{\text {); }}$ para lo cual, se analizaron las 48 observaciones que conforman el período de análisis. Es así, que se evidencia un coeficiente de correlaci66.1\% durante el período analizado, con lo cual se verifica la relación positiva entre el crecimiento de la economía y el desarrollo del sistema financiero, expresado como la expansión de la cartera. En ésta línea, Goldsmith citado por Dias (2014), estudia ésta relación en 35 países en el período 1860 a 1963, concluyendo que el crecimiento de los servicios financieros es una respuesta a una demanda originada en el desarrollo de la economía, efecto que se verifica en la economía ecuatoriana para el período estudiado.

\section{TABLA 1}

REGRESIÓN LINEAL PARA LA VARIACIÓN INTERANUAL DEL SALDO TOTAL DE LA CARTERA DE LOS BANCOS CONFORMANTES DEL SISTEMA FINANCIERO ECUATORIANO A PARTIR DE LA VARIACIÓN INTERANUAL DEL P.I.B. 2004-2016

\begin{tabular}{|c|c|c|c|c|c|}
\hline \multirow[t]{2}{*}{ Modelo } & \multicolumn{2}{|c|}{$\begin{array}{l}\text { Coeficientes no } \\
\text { estandarizados }\end{array}$} & \multirow{2}{*}{$\begin{array}{c}\text { Coeficien } \\
\text { tes } \\
\text { tipificado } \\
\text { s } \\
\text { Beta }\end{array}$} & \multirow[t]{2}{*}{$\mathrm{t}$} & \multirow[t]{2}{*}{ Sig. } \\
\hline & B & Error típ. & & & \\
\hline Constante & 0,066 & 0,018 & & 3,631 & 0,001 \\
\hline VARPIB & 0,956 & 0,158 & 0,661 & 6,040 & 0,000 \\
\hline
\end{tabular}

R2 ajustado=0,425; Durbin-Watson=0,472; Anova del modelo $\mathrm{F}=36,486$ (1gl, Sig. $=0,000)$.

Fuente: Elaboración propia en base a la información del Superintendente de Bancos del Ecuador y Banco Central del Ecuador

Aunque el propósito de nuestro análisis fue evidenciar el sentido de la relación entre VAR $_{\text {CAR y }} \mathrm{VAR}_{\mathrm{PIB}}$, es importante reconocer en los datos presentados, que al analizar la dispersión de los datos en relación a la línea de regresión mediante, mediante el coeficiente de determinación ( $\mathrm{R} 2=0,425)$, la fluctuación del PIB explica un $42.50 \%$ de las variaciones en los saldos de la cartera de los bancos que conforman el Sistema Financiera Ecuatoriano, dado que la $\mathrm{VAR}_{\mathrm{PIB}}$ es importante como variable explicativa del crecimiento de la cartera existen otros factores que inciden en su nivel.

Por otro lado, al aplicar la suma de las diferencias al cuadrado de los residuos sucesivos sobre el modelo de regresión, según la Prueba de Durbin-Watson a los 48 datos que conforman la serie de tiempo analizada, se obtiene un valor del estadístico de 0,472 , que al ser 
comparado con los límites establecidos para el caso propuesto $\left(\mathrm{d}_{1}=1.503\right.$ y du$\left.=1.585\right)$, se concluye que no se puede rechazar la hipótesis de que hay correlación serial positiva en los residuos, siendo una explicación del fenómeno la inercia propia de las series de tiempo económica, donde a partir del fondo de los periodos de recesión, en el período de recuperación, la mayoría de éstas series empiezan a moverse hacia arriba generándose un "impulso" en éstas que continuará hasta el próximo suceso que provoque un cambio de tendencia (Gujarati \& Porter, 2009).

En las Tabla 2 se resume el modelo que relaciona la variación interanual del índice de morosidad promedio de los bancos conformantes del sistema financiero ecuatoriano $\left(\mathrm{VAR}_{\mathrm{MOR}}\right)$ con la variación interanual del PIB de la economía nacional en los diferentes trimestres analizados (VAR $\mathrm{V}_{\mathrm{PIB}}$ ); aunque persiste el problema de un bajo coeficiente de determinación y auto correlación en los datos, se confirma los resultados de trabajos previos (Aguilar, G., Camargo, G. y Morales, R. 2006; Farhat, S, 2015; Olvera, A, 2010), pues es evidente la relación inversa entre estas dos variables analizadas en el periodo especificado

TABLA 2

REGRESIÓN LINEAL PARA LA VARIACIÓN INTERANUAL DEL ÍNDICE DE MOROSIDAD PROMEDIO DE LOS BANCOS CONFORMANTES DEL SISTEMA FINANCIERO ECUATORIANO A PARTIR DE LA VARIACIÓN INTERANUAL DEL P.I.B. 2004-2016

\begin{tabular}{|l|r|r|r|r|r|}
\hline Modelo & \multicolumn{2}{|c|}{$\begin{array}{c}\text { Coeficientes no } \\
\text { estandarizados }\end{array}$} & $\begin{array}{c}\text { Coeficientes } \\
\text { tipificados }\end{array}$ & $\mathrm{t}$ & \multicolumn{2}{c|}{ Sig. } \\
\cline { 2 - 7 } & $\mathrm{B}$ & Error típ. & Beta & & 0,001 \\
\hline $\begin{array}{l}\text { (Constant } \\
\text { e) }\end{array}$ & 0,119 & 0,032 & & 3,722 & 0,000 \\
VARPIB & $-1,709$ & 0,279 & $-0,666$ & $-6,129$ & \\
\hline
\end{tabular}

R2 ajustado=0,432; Durbin-Watson=0,395; Anova del modelo F=37.559 (1gl, Sig.=0,000).

Fuente: Elaboración propia en base a la información del Superintendente de Bancos del Ecuador y Banco Central del Ecuador

Con los resultados expuestos hasta este momento, es posible puntualizar que en el caso ecuatoriano se confirma la expectativa teórica, donde el ciclo económico se correlaciona de forma positiva con el saldo de la cartera de los bancos y de forma inversa con el índice de morosidad presentado por los bancos en el período estudiado.

\section{Análisis de la provisión para créditos incobrables realizadas en el sistema bancario en el período $2004-2016$}

Continuando con el análisis, se estudió la correlación entre la variación de la cuenta

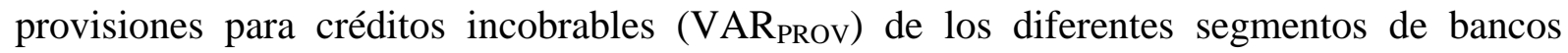
ecuatorianos como variable dependiente y como variables explicativas del modelo se utilizó la tasa variación anual del producto interno bruto expresado en su resultado trimestral (VAR $\left.\mathrm{VIB}_{\mathrm{B}}\right)$, la variación interanual de los saldos de cartera al final de cada trimestre $\left(\mathrm{VAR}_{\mathrm{CAR}}\right)$ y la variación del índice de morosidad ( $\left.\mathrm{VAR}_{\mathrm{MOR}}\right)$. Los resultados del análisis de correlación de Pearson se muestran en la Tabla 3, donde se evidencia una correlación positiva entre las variables estudiadas y la $\mathrm{VAR}_{\mathrm{PROV}}$; aunque, es importante anotar que únicamente el valor de probabilidad asociado para el coeficiente de correlación de la $\mathrm{VAR}_{\mathrm{MOR}}$ es menor 0.05 y por tanto se puede afirmar que la correlación es significativa, en este caso. 
TABLA 3

CORRELACIÓN DE LA VARIACIÓN INTERANUAL DE LA CUENTA PROVISIONES PARA CRÉDITOS INCOBRABLES CON LA VARIACIÓN DEL ÍNDICE DE MOROSIDAD, SALDOS DE CARTERA Y EL P.I.B. 2004-2016.

\begin{tabular}{|l|c|c|c|}
\hline & VAR $_{\text {PIB }}$ & VAR $_{\text {CAR }}$ & VAR $_{\text {MOR }}$ \\
\hline $\begin{array}{l}\text { Correlación de } \\
\text { Pearson }\end{array}$ & 0.012 & 0.118 & 0.341 \\
\hline Sig. (unilateral) & 0.468 & 0.210 & $0.008 * *$ \\
\hline $\mathrm{N}$ & 49 & 49 & 49 \\
\hline
\end{tabular}

** La correlación es significativa el nivel de 0.01 (unilateral)

Fuente: Elaboración propia en base a la información del Superintendente de Bancos del Ecuador y Banco Central del Ecuador

Los datos resumidos en la tabla precedente confirman los estudios previos sobre el tema, donde se pone en evidencia que en el análisis del riesgo de crédito que realizan los bancos al evaluar la capacidad de pago de sus clientes prima el examen retrospectivo del cumplimiento las obligaciones de éstos sobre sus expectativas futuras, expresado en el nivel de morosidad. Se evidencia también la baja significancia tanto de la VAR $\mathrm{PIB}_{\text {como de la }}$ VAR $_{\text {CAR }}$ como variables explicativas de la variación de la cuenta provisiones.

Para evidenciar de manera más efectiva el efecto descrito y muy importante evaluar los resultados de la provisión anticíclica, se optó por dividir el período de análisis en dos sub períodos: uno anterior a la vigencia de la norma, es decir para los períodos precedentes al tercer trimestre del año 2012 y otro para el período subsiguiente.

\section{Efecto de la provisión anticíclica en la estructura de las provisiones para cuentas incobrables}

En la Tabla 4 se exponen los resultados del análisis correlacional en el primer sub-período planteado; donde se advierte la existencia de una correlación directa significativa entre la $\mathrm{VAR}_{\mathrm{MOR}}$ y VAR $\mathrm{VAR}_{\mathrm{PRO}}$ al $65.7 \%$, es decir que mientras se evidencie un crecimiento del índice de morosidad producto de la mora en el pago de los créditos, las provisiones tenderán a subir como su efecto inmediato. Por otro lado, se destaca la existencia de una correlación inversa entre $\mathrm{VAR}_{\mathrm{PIB}}$ y $\mathrm{VAR}_{\mathrm{PROV}}$ igual al $36.4 \%$, con lo cual se confirma la relación contra-cíclica, explicada en el marco teórico, entre $\mathrm{VAR}_{\mathrm{PROV}}$ y $\mathrm{VAR}_{\mathrm{PIB}}$ para el sub-periodo analizado. En ambos casos se advierte la asociación significativa entre las variables, por tal razón se plantea la posibilidad de un modelo de regresión multinomial. Finalmente, como ya se destacó en líneas anteriores la $\mathrm{VAR}_{\mathrm{PIB}} \mathrm{y} \mathrm{VAR}_{\mathrm{CAR}}$ tienen una fuerte correlación positiva, por lo que es congruente la relación inversa entre ésta última variable y la variación de las provisiones para éste sub-período de estudio.

\section{TABLA 4}

CORRELACIÓN DE LA VARIACIÓN INTERANUAL DE LA CUENTA PROVISIONES PARA CRÉDITOS INCOBRABLES CON LA VARIACIÓN DEL ÍNDICE DE MOROSIDAD, SALDO DE CARTERA TOTAL Y EL P.I.B. 2004- $2^{\circ}$ TRMESTRE 2012

\begin{tabular}{|l|c|c|c|}
\hline & $\mathrm{VAR}_{\mathrm{PIB}}$ & $\mathrm{VAR}_{\mathrm{CAR}}$ & $\mathrm{VAR}_{\mathrm{MOR}}$ \\
\hline $\begin{array}{l}\text { Correlación de } \\
\text { Pearson }\end{array}$ & $-0.364^{*}$ & -0.263 & $0.657^{* *}$ \\
\hline Sig. (unilateral) & 0.022 & 0.076 & 0.000 \\
\hline
\end{tabular}




\begin{tabular}{|l|l|l|l|}
\hline $\mathrm{N}$ & 31 & 31 & 31 \\
\hline
\end{tabular}

** La correlación es significativa el nivel de 0.01 (unilateral)

Fuente: Elaboración propia en base a la información del Superintendente de Bancos del Ecuador y Banco Central del Ecuador

Luego, al estructurar el correspondiente modelo de regresión y tomando como variable dependiente a la variación de la cuenta provisiones, se advierte un escenario de predicción equivalente al $45.5 \%$ ( $\mathrm{r} 2$ ajustado 0,455), sin embargo, el modelo muestra que existen dependencias de residuos (Durbin Watson inferior a 1), así como una predicción de P.I.B. que no favorece al modelo. En tal sentido, se demuestra que, el PIB no es portador de explicación de la cuenta provisiones en el período anteriormente al segundo trimestre del año 2012, siendo importante subrayar que para el periodo estudiado la variación cuenta de morosidad se destaca como variable explicativa de la variación de las provisiones para créditos incobrables (ver Tabla 5).

TABLA 5

REGRESIÓN LINEAL MÚLTIPLE PARA LA CUENTA PROVISIONES PARA CRÉDITOS INCOBRABLES A PARTIR DE LA VARIACIÓN DEL ÍNDICE DE MOROSIDAD, SALDO DE CARTERA Y EL P.I.B. 2004-2 TRMESTRE 2012

\begin{tabular}{|l|c|c|c|c|l|}
\hline & B & Error estándar & $\begin{array}{c}\text { Coeficientes } \\
\text { estandarizados } \\
\text { Beta }\end{array}$ & $\mathrm{t}$ & \multicolumn{1}{|c|}{ Sig. } \\
\hline Constante & 0.133 & 0.034 & & 3.955 & $0,000 * * *$ \\
\hline VAR $_{\text {CAR }}$ & 0.340 & 0.172 & 0.368 & 1.978 & 0,058 \\
\hline VAR $_{\text {MOR }}$ & 0.442 & 0.099 & 0.852 & 4.449 & $0,000 * * *$ \\
\hline VAR $_{\text {PIB }}$ & -0.172 & 0.206 & -0.127 & -0.835 & 0.411 \\
\hline
\end{tabular}

R2 ajustado=0,455; Durbin-Watson=0,420; Anova del modelo F=9.337 (2gl, Sig.=0,000); Media de los FIV=1,73.

Fuente: Elaboración propia en base a la información del Superintendente de Bancos del Ecuador y Banco Central del Ecuador

Una vez que se ha definido las relaciones entre las variables para el primer subperíodo de análisis, se procede a estudiar la forma como se relacionan las variables estudiadas y como las influyen cada una de éstas en la variación de los saldos de la cuenta provisiones, en el segundo sub-período de análisis propuesto. Cabe destacas, que en éste segundo escenario, se evidencia cambios significativos; pues, en lo que respecta $V_{A R} R_{P I B}$ se advierte que deja de correlacionarse inversamente y pasa a generar un coeficiente positivo del $54.4 \%$ (ver Tabla 6); con lo cual, se evidencia el efecto de la aplicación de la normativa en la que se obliga a las instituciones financiera a constituir provisiones anticíclicas, es decir que mientras se experimente etapas de expansión económica se provisione para respaldar al sistema financiero en el caso de que se concreten altos índices de morosidad en futuras fases de recesión de la economía.

\section{TABLA 6}

CORRELACIÓN DE LA VARIACIÓN INTERANUAL DE LA CUENTA PROVISIONES PARA CRÉDITOS INCOBRABLES CON LA VARIACIÓN DEL ÍNDICE DE MOROSIDAD, SALDO DE CARTERA Y EL P.I.B: $3^{\circ}$ TRIMESTRE 2012-2016

\begin{tabular}{|l|c|c|c|}
\hline & VAR $_{\mathrm{PIB}}$ & $\mathrm{VAR}_{\mathrm{CAR}}$ & $\mathrm{VAR}_{\mathrm{MOR}}$ \\
\hline $\begin{array}{l}\text { Correlación de } \\
\text { Pearson }\end{array}$ & $0.544^{*}$ & $0.754^{* *}$ & 0.111 \\
\hline
\end{tabular}




\begin{tabular}{|l|c|c|c|}
\hline Sig. (unilateral) & 0.020 & 0.000 & 0.331 \\
\hline $\mathrm{N}$ & 18 & 18 & 18 \\
\hline
\end{tabular}

* La correlación es significativa el nivel de 0.1 (unilateral)

** La correlación es significativa el nivel de 0.01 (unilateral)

Fuente: Elaboración propia en base a la información del Superintendente de Bancos del Ecuador y Banco Central del Ecuador

Otro punto importante a destacar es la alta correlación positiva $(75.4 \%)$ que tiene la $\mathrm{VAR}_{\mathrm{CAR}}$ y la estructura de las provisiones, con lo cual se pone en evidencia el efecto que ejerce los cambios en la normativa sobre la estructura de las provisiones. Es así, que el cálculo de la provisión anticíclica (1) parte del valor calculado de la pérdida latente (2); por consiguiente, dado el aumento de los saldos de la cartera crediticia de los bancos, se esperaría un aumento de dicha pérdida latente y por ende mayores niveles de provisiones para cuentas incobrables, relación que se confirma con el presente estudio. A continuación, se expresa el modelo de regresión lineal múltiple:

\section{TABLA 7}

REGRESIÓN LINEAL MÚLTIPLE PARA LA CUENTA PROVISIONES PARA CRÉDITOS INCOBRABLES A PARTIR DE LA VARIACIÓN DEL ÍNDICE DE MOROSIDAD, SALDO DE CARTERA Y EL P.I.B: $3^{\circ}$ TRIMESTRE 2012-2016

\begin{tabular}{|l|c|c|c|c|l|}
\hline & B & Error estándar & $\begin{array}{c}\text { Coeficientes } \\
\text { estandarizados } \\
\text { Beta }\end{array}$ & $\mathrm{t}$ & \multicolumn{1}{|c|}{ Sig. } \\
\hline Constante & -0.366 & 0.021 & & -1.701 & 0.111 \\
\hline VAR $_{\mathrm{CAR}}$ & 0.771 & 0.193 & 0.659 & 3.993 & $0.001^{* *}$ \\
\hline VAR $_{\mathrm{MOR}}$ & 0.554 & 0.109 & 0.711 & 5.075 & $0.000^{* * *}$ \\
\hline VAR $_{\mathrm{PIB}}$ & 0.815 & 0.322 & 0.504 & 2.531 & $0.024^{*}$ \\
\hline
\end{tabular}

R2 ajustado=0,816; Durbin-Watson=1.004; Anova del modelo F=26.201 (3gl, Sig.=0,000); Media de los FIV=2.66.

Fuente: Elaboración propia en base a la información del Superintendente de Bancos del Ecuador y Banco Central del Ecuador

Al hacer el análisis del modelo, se advierte una predictibilidad mayor, equivalente al $81.6 \%$ (r2 ajustado 0,816), en ella se observa que el cumplimiento de todos los supuestos, incluida la independencia de los residuos (próxima a 2). En efecto, a partir del tercer trimestre del año 2012, la VARMOR y VARCAR convergen como generadoras de explicaciones de manera significativa, a pesar de que el valor de la constante disminuye su nivel de explicación. Es muy importante destacar el problema de multicolinealidad que afecta al modelo, pues como ya se anotó las variables explicativas tienen estrecha relación, y donde según el análisis del factor de inflación de la varianza (FIV) la variable más afecta es la VARPIB dada sus relaciones ya explicadas previamente en este trabajo.

Con los datos expuestos, se demuestra, por lo tanto, que el $\mathrm{VAR}_{\mathrm{PIB}} \mathrm{y}$ principalmente la $\mathrm{VAR}_{\mathrm{CAR}}$, tras la implementación de la obligatoriedad de estructurar las provisiones anticíclicas, adquiere una gran significancia como variable explicativa dentro de la variación de la cuenta provisiones para créditos incobrables. Por lo tanto, es posible anotar que, a partir de la emisión de la correspondiente resolución, el análisis del ciclo económico adquiere un valor preponderante para la estructura de provisiones, expresada a través del concepto de la pérdida latente, el cual se consolida como un factor importante en la estructura de las 
provisiones para créditos incobrables de los bancos conformantes del sistema financiero ecuatoriano a partir del tercer trimestre del año 2012.

\section{CONCLUSIONES}

Es importante, luego del presente estudio, destacar el efecto que ejerce el ciclo económico en el sector financiero nacional ecuatoriano y más concretamente sobre la estructura de la provisiones para créditos incobrables; dado que, en primera instancia, se resalta la correlación positiva entre la variación de la cartera de los bancos y el crecimiento de la económica nacional; pues, en este trabajo se pone en evidencia que en épocas expansivas de la economía ecuatoriana, los saldos de la cartera de créditos de los bancos crece, por efecto del aumento de la demanda de créditos y el aumento de ahorro de los hogares y empresas. Por lo expuesto, se puntualiza que al crecer los saldos de cartera crece el riesgo de que los prestatarios caigan en mora lo cual en la codificación pertinente se identifica bajo el concepto de pérdida latente.

Otro resultado a destacar es la relación inversa entre el PIB y el índice de morosidad de los bancos de nuestro país; donde, se pone de manifiesto el hecho de que, a un mayor crecimiento económico, mejora la capacidad de pago de los deudores de los bancos reduciéndose las tasas de morosidad crediticia.

En el marco de los resultados antes expuestos se destaca que, como consecuencia del crecimiento económico existirá un efecto en la evaluación del riesgo crediticio y por ende en la estructura de las provisiones para créditos incobrables; bien sea por el incremento de la cartera bruta y por ende de la perdida latente calculada o por la variación del índice de morosidad y la consiguiente calificación del riesgo en las diferentes operaciones crediticias.

En el transcurso de nuestra investigación se identificó que, en la estructura de las provisiones, por mandato normativo, se obliga a partir de tercer trimestre de 2012 incluir la provisión anticíclica, como una respuesta ante el efecto que tiene el ciclo económico en la estructura de las provisiones. Luego de analizar la incidencia de las variaciones del PIB sobre la variación de las provisiones para cuentas incobrables en el periodo 2004-2016, es importante dividir el período estudiado y distinguir sus particularidades y más aún la explicación de estas diferencias.

En un primer análisis, se posible puntualizar que en los años precedentes al 2012 el sistema bancario presenta una correlación negativa entre la VAR $\mathrm{VRRV}_{\text {y }}$ la VAR $\mathrm{V}_{\mathrm{PIB}}$ y por otro lado una relación positiva entre la $\mathrm{VARP}_{\mathrm{ROV}}$ y la $\mathrm{VAR}_{\mathrm{MOR}}$, con lo cual se evidencia que las instituciones financieras estructuran sus provisiones bajo análisis retrospectivo del cliente y su capacidad para honrar sus deudas, lo cual se pone de manifiesto en la calidad de la cartera por el índice de morosidad; donde los bancos al sufrir un incremento de la morosidad de su cartera suben sus niveles de provisiones y viceversa. En este mismo análisis, se observó que los bancos al percibir mejoradas las condiciones de pago de sus clientes por las condiciones favorables del ambiente de crecimiento económico, como ya se anotó, reducen sus provisiones para cuentas incobrables, con lo cual se explica la correlación negativa entre la variación interanual de estas dos variables.

Prosiguiendo con ésta síntesis, se puede destacar que el año 2012 con la inclusión, por poder normativo, de las provisiones anticíclicas, se corrige el efecto del ciclo económico; pues, en el análisis correlacional se evidencia que para el periodo 2012 -2016 la VAR PROv y la $\mathrm{VAR}_{\text {PIB }}$ cambian su correlación y se aprecia una relación positiva. Esta correlación positiva es el resultado del incremento de la provisión anticíclica, la cual en épocas de auge económico se 
acumula y en épocas de depresión económica, cuando se materializa el incumplimiento en la cartera, suple un efecto compensatorio a las posibles deficiencias provisionales.

Por lo expuesto consideramos que es evidente el efecto de la provisión anticíclica en la estructura de las provisiones para créditos incobrables, con lo cual el sistema bancario ecuatoriano, bajo el punto de vista del efecto del ciclo económico, cuenta con provisiones para afrontar posibles futuras épocas de depresión económica, a la par que se puede puntualizar que los estados financieros presentan de una forma más efectiva la situación real de sus activos.

Es importante anotar que el presente estudio se concentra en el análisis de las provisiones bajo el efecto de las fluctuaciones del PIB; por lo cual, será importante complementar éste análisis, a fin de evaluar el efecto de otras variables bien sean endógenas o exógenas al sistema financiero y determinar en qué grado afectan a la eficiencia de la estructura de las provisiones para créditos incobrables de los bancos ecuatorianos.

\section{Referencias}

Aguilar, G., Camargo, G. y Morales, R. (2006). Análisis de la Morosidad en el Sistema Bancario Peruano. Economía y sociedad. (62), 74-81. Recuperado de http://cies.org.pe/sites/default/files/files/otros/economiaysociedad/10_aguilar.pdf

Alfaro, R., Calvo, D., \& Oda, D. (2009). Riesgo de Crédito de la Banca de Consumo. Economía Chilena, 12(3), 59-77. Recuperado de https://www.researchgate.net/profile/Rodrigo_Alfaro/publication/46529841_Consume r_Banking_and_Credit_Risk/links/00b7d528a22f03f91c000000/Consumer-Bankingand-Credit-Risk.pdf

Asamblea Constituyente. (2008) Constitución de la República del Ecuador. Publicado en Registro Oficial 449, de 20-oct-2008. Quito, Ecuador.

Asamblea Nacional. (2014). Código Orgánico Monetario y Financiero. Publicado en Registro Oficial No 332, de 12-sept-2014. Quito, Ecuador.

Banco Central del Ecuador. (2017). Cuentas nacionales trimestrales del Ecuador (Boletín No.96). Recuperado de: https://contenido.bce.fin.ec/home1/estadisticas/cntrimestral/CNTrimestral.jsp

Bushman, R. M., \& Williams, C. D. (2012). Accounting discretion, loan loss provisioning, and discipline of Banks' risk-taking. Journal of Accounting and Economics, 54(1), 118. http://dx.doi.org/10.2139/ssrn.1521584

Dias, V. (2014). Crédito privado, crédito bancario y producto interno bruto: evidencia para una muestra suramericana. Ensayos Sobre Politica Económica, 32(73), 104-126. https://doi.org/10.1016/j.espe.2015.09.001

Gujarati, D \& Porter, D. (2009). Econometría. México: McGraw Hill.

Farhat, S. D. (2015). La Probabilidad De Incumplimiento De Los Hogares, ¿ Mito o Realidad?. Observatorio de la Economía Latinoamericana, (214). Recuperado de: http://www.eumed.net/cursecon/ecolat/ec/2015/hogares.html 
Freire, M. (2009). Sorpresas en el análisis de la liquidez, el crédito y la regulación estatal. Koyuntura - Revista del Instituto de Economía USFQ, 5(5), 1-8. Recuperado de http://www.usfq.edu.ec/publicaciones/koyuntura/Documents/koyuntura_005.pdf

Gonzalez, C. O. (2015). La produccion y el desempleo una relación o simple coincidencia ley de okun para el ecuador. Ciencia Unemi, 1(2), 42-47. Recuperado de http://ojs.unemi.edu.ec/index.php/cienciaunemi/article/view/146

Hilbers, P., Otker-Robe, I., \& Pazarbasioglu, C. (2006). ¿Velocidad excesiva?: cómo hacer frente al rápido crecimiento del crédito en europa Central y orientaL. Finanzas y desarrollo: publicación trimestral del Fondo Monetario Internacional y del Banco Mundial, 43(1), 42-45. Recuperado de http://www.imf.org/external/pubs/ft/fandd/spa/2006/03/pdf/hilbers.pdf

Jara, A. (2005). Provisiones bancarias y ciclo económico: el caso de Chile. Informe de Estabilidad Financiera (Primer Semestre) Banco Central de Chile. Recuperado de https://www.researchgate.net/publication/242692478_Provisiones_bancarias_y_ciclo_ economico_el_caso_de_Chile

Novales, A. (2010). Política monetaria antes y después de la crisis financiera. Claves de la economía mundial, 10(), 2011, 29-40. Recuperado de https://www.ucm.es/data/cont/docs/518-2013-11-15-Crisis.pdf

Pita Fenández, S., \& Pértega Díaz, S. (1997). Relación entre variables cuantitativas. Cad Aten Primaria, (4), 141-144. Recuperado de http://prod.fisterra.com/mbe/investiga/var_cuantitativas/var_cuantitativas2.pdf

Olvera, A. (2010). Supervisión bancaria y crisis financiera. El caso español. Revista Encontros Científicos. (6), 146-161. Recuperado de http://www.scielo.mec.pt/scielo.php?script=sci_arttext\&pid=S164624082010000100014

Ruiz, F. (2008). Provisiones y contingencias. AECA: Revista de La Asociación Española de Contabilidad Y Administración de Empresas, (81), 58-63. Recuperado de http://www.aeca1.org/revistaeca/revista81/81.pdf

Salas, J. S. (2002). Solvencia bancaria, riesgo de crédito y regulación pública: el caso de la provisión estadística española. Revista de Economía Pública. 161(2), 129-150. Recuperado de http://www.ief.es/documentos/recursos/publicaciones/revistas/hac_pub/161_solvencia s.pdf

Sánchez, M. (2008). Determinar provisiones anticíclicas en cartera de crédito, para mantener la solvencia en entidades bancarias del sistema financiero ecuatoriano. (Tesis de maestría) Universidad Andina Simón Bolívar Sede Ecuador. Quito, Ecuador

Seffino, M., \& Hoyos Maldonado, D. (2016). Eficiencia bancaria en Argentina. Comportamiento de los bancos entre 2005 y 2013. Estudios Gerenciales, 32(138), 44 50. https://doi.org/10.1016/j.estger.2015.12.002 
Superintendencia de Bancos (2017). Boletines mensuales bancos privados. Recuperado de http://www.superbancos.gob.ec/practg/sbs_index?vp_art_id=5036\&vp_tip=2\&vp_bus $\mathrm{cr}=41$

Superintendencia de Bancos (2016). Codificación Resoluciones SB y Junta Bancaria (Libro 1.- Normas generales para las instituciones del sistema financiero, Titulo IX: De los activos y de los límites de los créditos). Recuperado de http://www.superbancos.gob.ec/medios/PORTALDOCS/downloads/normativa/nueva_ codificacion/todos/L1_IX_cap_II.pdf

Superintendencia de Bancos (2016). Codificación Resoluciones SB y Junta Bancaria (Libro 1.- Normas generales para las instituciones del sistema financiero, Título X: De la gestión y administración de riesgo). Recuperado de http://www.superbancos.gob.ec/medios/PORTALDOCS/downloads/normativa/nueva_ codificacion/todos/L1_IX_cap_II.pdf)

Superintendencia de Bancos (2016). Plan de cuentas y catálogo. Recuperado de http://www.superbancos.gob.ec/medios/PORTALDOCS/downloads/normativa/CUC/ CUC_plan_cuentas_activos_2_dic_15.pdf

Superintendencia de Bancos del Ecuador. (2012). Resolución No JB-2012-2217. Recuperado de:

http://www.superbancos.gob.ec/medios/PORTALDOCS/downloads/normativa/2012/r esol_JB-2012-2217.pdf

Superintendencia de Bancos del Ecuador. (2012). Circular No.SBS-DNR-INSFPR-2012-7570. Recuperado http://www.superbancos.gob.ec/medios/PORTALDOCS/downloads/normativa/2012/r esol_JB-2012-2217.pdf

Zepeda, E., Leos, J. A., \& Carvallo, F. (2016). Capital social y mercados financieros crediticios: demanda de crédito en México, 2010. Problemas del Desarrollo. 47(184), 61-85. https://doi.org/10.1016/j.rpd.2016.01.004

ZHEN-JIA-LIU. (2015). Cross-country study on the determinants of bank financial distress. Revista de Administração de Empresas, 55(5), 593-603. doi:10.1590/S0034759020150510 